\title{
THE DETERMINATION OF ACCURACY OF THE DEMONSTRATOR OF AERONAUTIC BEVEL GEARBOX, ACCOMPLISHED BY SELECTED RAPID PROTOTYPING TECHNIQUES USING AN OPTICAL SCANNER ATOS II TRIPLE SCAN
}

\author{
Adam Marciniec, Grzegorz Budzik \\ Tomasz Dziubek, Bartlomiej Sobolewski, Małgorzata Zaborniak \\ Rzeszow University of Technology \\ Faculty of Mechanical Engineering and Aeronautic \\ Powstancow Warszawy Av. 8, 35-959 Rzeszow, Poland \\ e-mail:amarc@prz.edu.pl,gbudzik@prz.edu.pl \\ tdziubek@prz.edu.pl,b_sobolewski@prz.edu.pl,mzab@prz.edu.pl
}

\begin{abstract}
Designing and manufacturing of aeronautic bevel gearboxes is a complicated and time-consuming process because of complex kinematics of the machining process as well as numbers of manufacturing methods. Machine manufacturers usually provide algorithms used in manufacture process. This requires substantial financial resources and, in consequence, it increases the costs of production.

Using other commonly available calculation algorithm requires a lot of studies to verify whether the proposed gearbox works correctly. In order to reduce the manufacturing costs of prototypes, it is possible to use Rapid Prototyping methods. Using both Rapid Prototyping techniques and coordinate optical measurements enables to verify the correctness of assumptions made in the initial stage of product designing. This approach reduces significantly both prototyping time and manufacturing costs.

In order to ensure the effectiveness of prototyping process, it is essential to provide the accuracy of manufactured prototypes. That is the reason why it is necessary to determine manufacturing methods that will ensure the highest geometric accuracy.

The study presents the modelling and manufacturing process of aeronautic bevel gear of demonstrator taking into consideration the accuracy of selected Rapid Prototyping methods. The gear modelling is based on machining simulation method conducted in Autodesk Inventor. The measurement results are shown in displacement maps obtained with an optical scanner Atos II Triple Scan and universal GOM Inspect Professional software, which determines the prototype accuracy in relation to $3 D-C A D$ models.
\end{abstract}

Keywords: bevel gear, aircraft gearbox, optical scanner, coordinate measuring technique, Rapid Prototyping, gear modelling

\section{Introduction}

Despite continuous progress, bevel gears are still indispensable parts of drive systems. Therefore, bevel gears are widely used in many fields of the industrial practise; they are faced with many requirements. Using bevel gears in the aerospace industry is associated with specific requirements. Manufactured gearbox are required to transfer high loads while high reliability is maintained, weight reduced and noise emission limited. Tor this, reason, aviation industry uses spiral bevel gears. Bevel gear design process, for the aviation industry is complicated and difficult due to complex gear geometry, machining process kinematic and numerous manufacturing methods. Therefore, at the design stage it is necessary to verify if the gearbox operate properly. Gear quality indicator is the location, shape, contact pattern as well as evenly transmission error. To determine analysing tooth contact is necessary to carry out tests on the test stand, which involves the need to perform gears. This is time and costs consuming.

Progress, which has been made in the field of computer-aided design systems, allowed running 
virtual simulations bevel gear tooth contact. Nevertheless, it is still necessary to produce a prototype and examine it. Nowadays, additive manufacturing technologies are increasingly used that is why it was decided to examine the usefulness of Rapid Prototyping methods for the bevel gears prototypes production. The research aimed to determine the geometric accuracy of prototypes produced using different methods. The article presents the results obtained for the pinion.

\section{Preparing the prototypes}

The first stage of the research was making solid models of bevel gears in the CAD environment. Solid models of bevel gears were made using the method of solid machining simulation. Bevel gear machining simulation due to complex machining kinematics belongs to the most complicated cases of making bevel gear solid models in CAD environment $[2,4]$. Bevel gear model performed with SGT method by Gleason Company. Machining simulation is based on iterative execution of difference solids machined wheels and tools in subsequent positions arising from the machining kinematic. Way of creating solid model by machining simulation method presented on the example of the concave flank of the pinion tooth.

First, solid tools and blank of the workpiece are made and spaced on the basis of setup parameters. Then machining simulation process relies on an iterative execution of subtraction tools solid from workpiece solid, in positions resulting from the kinematic of the real machining process is carried out (Fig. 1).
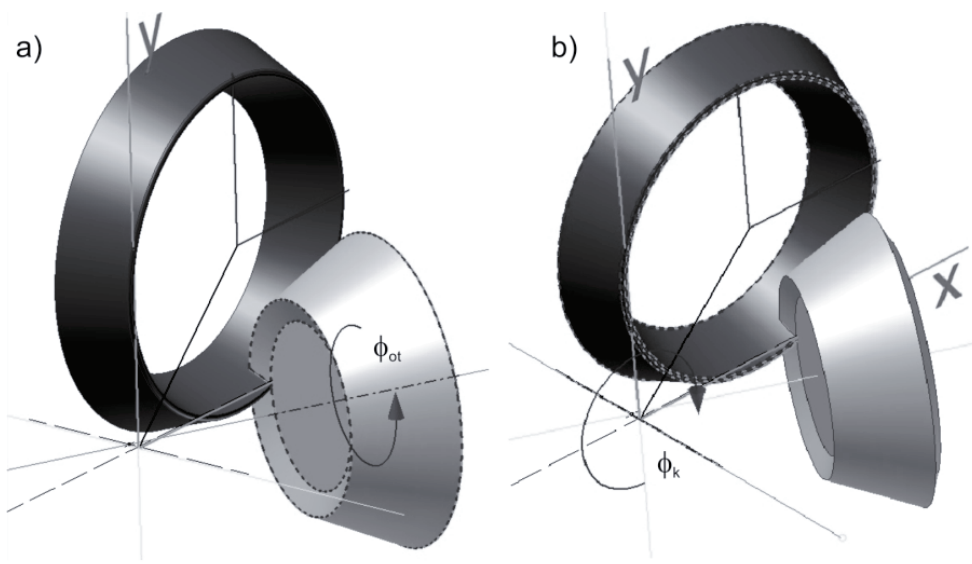

$\phi_{\mathrm{k}}=\mathrm{i}_{\text {odt }} \phi_{\text {ot }}$

Fig. 1. Machining simulation: a) blank discrete rotation, b) tool discrete rotation

Upon completion of the simulation obtained a concave pinion tooth flank (Fig. 2).
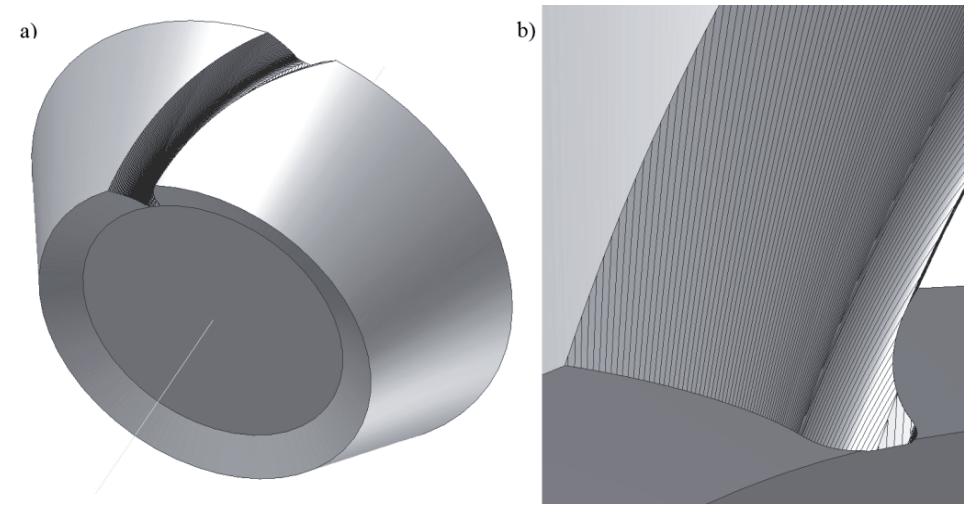

Fig. 2. Simulation results: a) received concave tooth flank, b) detailed view 
The same approach was used to determine the convex side of pinion tooth. As a result of submission, received solids obtained one notch. In order to simplify the procedure of the complete gear modelling, the notch surface, obtained in simulation result, replaced with a smooth surface. That surface was used to generate a solid model of the pinion (Fig. 3).

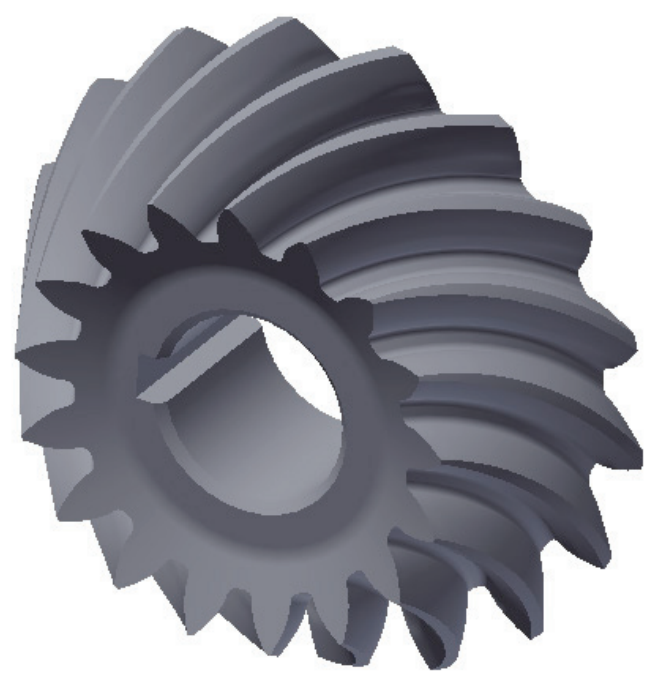

Fig. 3. Obtained pinion solid model

Then, on the basis of a solid model after exporting to a format appropriate for RP machines made prototypes using two methods of Rapid Prototyping: FDM and PolyJet.

\section{Determining prototypes accuracy}

To determine the accuracy of geometric received prototypes was performed using modern methods. Optical scanner was used to scan a light blue ATOS Triple Scan II. Using this tool allowed to gather information about the entire geometry of the analysed models. This made it possible to better define the accuracy and usefulness of Rapid Prototyping methods used in the research. After the measurement, performed an analysis of deviation-measured model compared to the 3D CAD model using the GUM Inspection program. The results obtained in the colour maps of global deviations. This was the basis for designate the deviations of tooth profile in selected cross-section [1,3]. The results obtained for the prototypes made by FDM method shows Fig. 4 and 5.

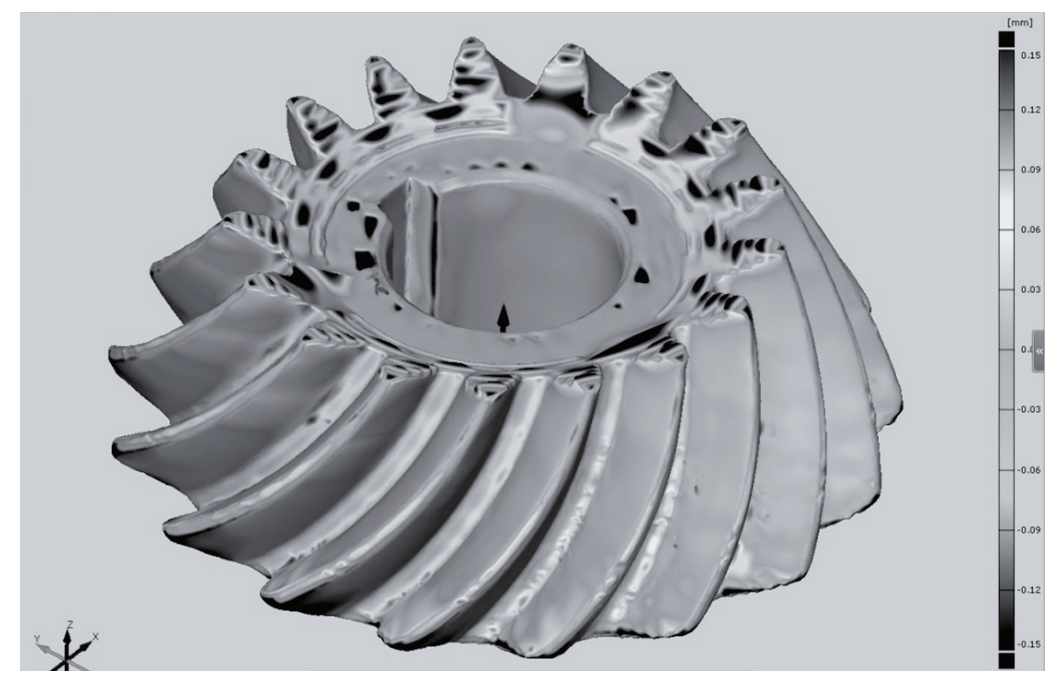

Fig. 4. deviations map obtained for the model made by FDM method 


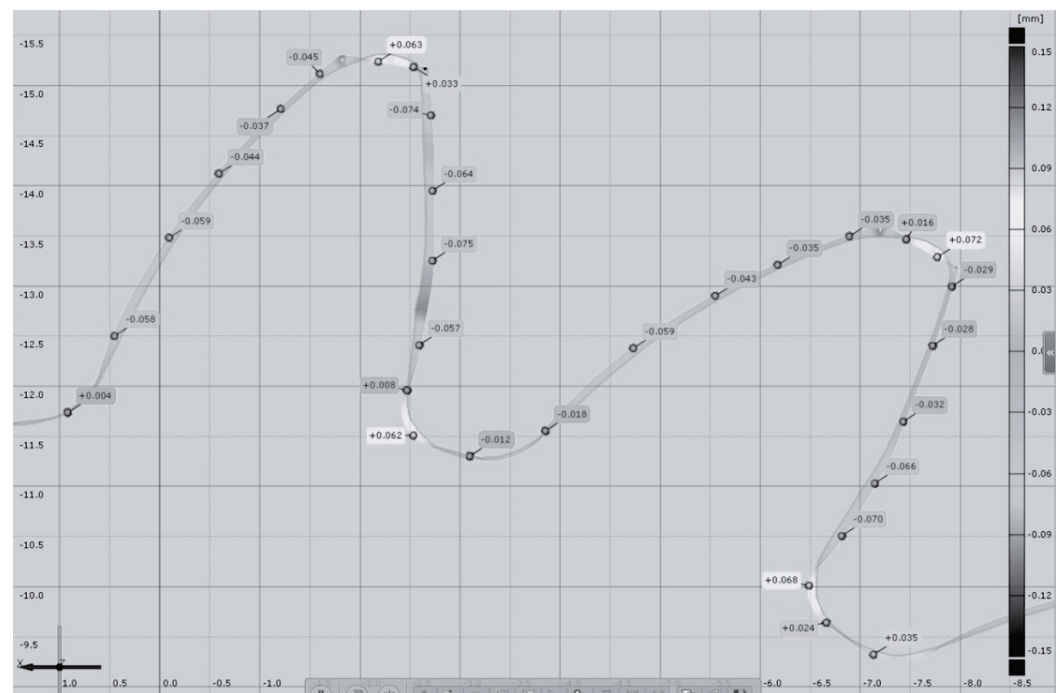

Fig. 5. deviations decomposition of the pinion tooth profile made FDM method

The analysis of obtained measurement results indicated that the tooth profile deviations are smaller than $0.1 \mathrm{~mm}$ and the highest values come to $0.15 \mathrm{~mm}$. Measurements and analyses for the prototype made by PolyJet method performed in the same way. Results show Fig. 6 and 7.

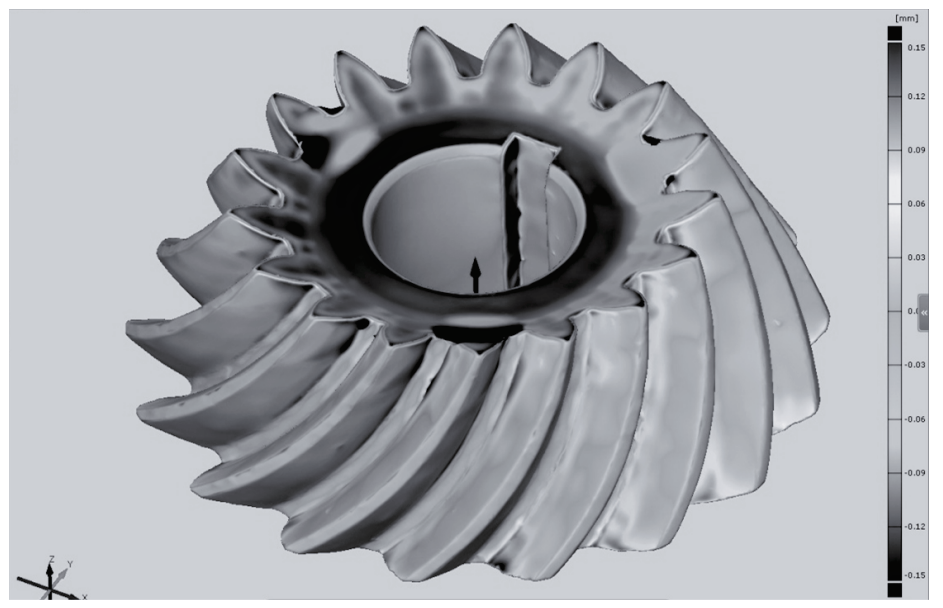

Fig. 6. deviations map obtained for the model made by PolyJet method

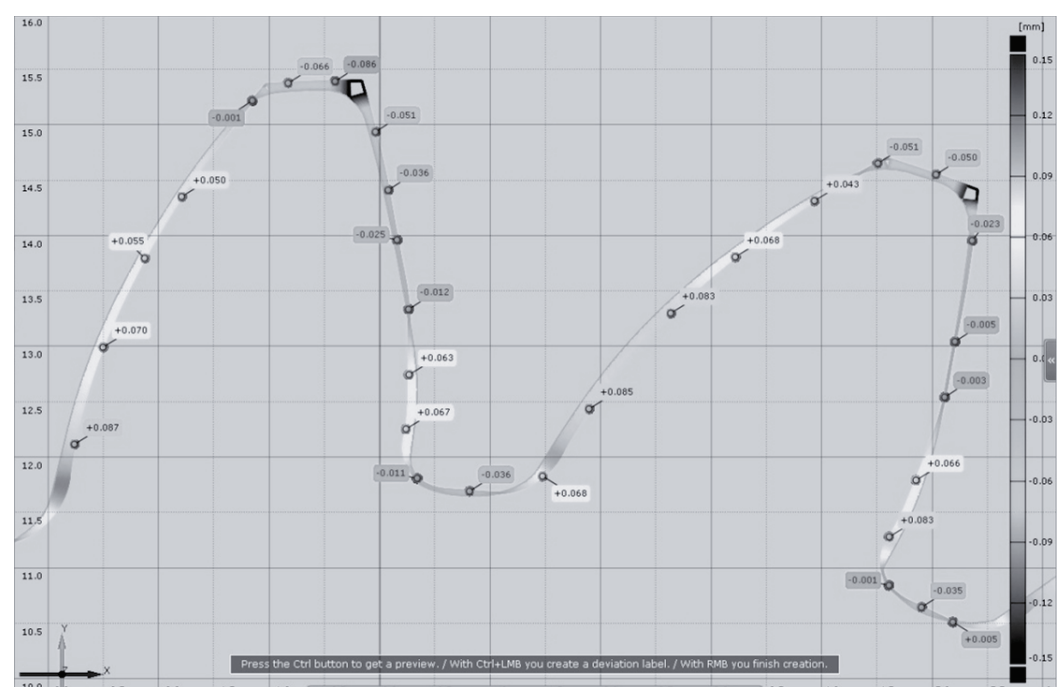

Fig. 7. Deviation decomposition of the pinion tooth profile made by PolyJet method 
In the case of using PolyJet, method to model manufacturing was observed similar values of deviations as in using FDM technology. It is however noted, that deviations measured on concave side pinion tooth are smaller.

\section{Conclusion}

This paper presents that using modern methods of manufacturing prototypes based on computer-aided design systems and Rapid Prototyping techniques enables production of real models with the accuracy of mapping the geometry about $0.1 \mathrm{~mm}$. On the basis of optical measurements and the results of the analysis it is possible to determine the size of modifying the geometry of manufactured prototypes aimed at overcoming the errors generated in Rapid Prototyping process. Their introduction will help to enhance the accuracy of mapping models, thus allow using Rapid Prototyping methods to manufacture prototypes for Tooth Contact Analysis.

\section{Acknowledgement}

Financial support of Structural Funds in the Operational Programme - Innovative Economy (IE OP) financed from the European Regional Development Fund - Project Modern material technologies in aerospace industry“, Nr POIG.01.01.02-00-015/08-00 is gratefully acknowledged.

\section{References}

[1] Kozik, B., Budzik, G., Sobolak, M., Dziubek, T., Sobolewski, B., Bernaczek, J., Grzelka, M., Accuracy analysis of demonstrative prototype of FDM model of aeronautical dual-power path gear unit, 4th International Scientific Conference - Management of Technology - Step to Sustainable Production - MOTSP 2012, Croatian Association for PLM, pp. 308-313, Zagreb, Croatia 2012.

[2] Marciniec, A., Sobolewski, B., Method of Spiral Bevel Gear Tooth Contact Analysis Performed in CAD Environment, 7th International Conference AIRTEC, Aircraft Engineering and Aerospace Technology, Vol. 85, Is. 6, 2012.

[3] Marciniec, A., Budzik, G., Dziubek, T., Grzelka, M., Quality control and inspection of bevel gears of the aircraft gearbox utylizing the ATOS 3D Scanner, Journal of KONES Powertrain and Transport, Vol. 19.

[4] Skawiński, P., Integration of Design and Manufacturing of Spiral and Hypoid Bevel Gears, Mechanika 236, Warsaw 2010. 